\title{
Measurement of involuntary arm movement in athetotic patients
}

\author{
PETER D. NEILSON ${ }^{1}$ \\ From the Division of Neurology, The Prince Henry Hospital, and the Schools \\ of Medicine and Physics, University of New South Wales, Sydney, Australia
}

SYNOPSIS Visual tracking tests have been used to obtain a quantified statistical description of the involuntary movements of the arm about the elbow joint in a group of patients suffering from athetoid cerebral palsy. Three separate components of involuntary activity can be recognized and it is possible that each may be a different physiological mechanism. First there are irregular movements which are represented by a continuous power spectrum which decreases with increasing frequency, reaching a negligible value between $2-3 \mathrm{~Hz}$. The second component is a rhythmical low frequency movement which is indicated by a predominant peak in the power spectrum at a frequency of 0.3 $0.6 \mathrm{~Hz}$. The presence of this peak was predicted in a previous paper because of underdamping demonstrated in the closed loop voluntary control of movement in athetosis. The third component is the athetoid action tremor in which both agonist and antagonist muscle groups contract vigorously but asynchronously at a frequency of $1 \cdot 5-4 \mathrm{~Hz}$.

Athetosis has been described as a disorder of the central nervous system which is manifested clinically by involuntary movements which lack fixed amplitude, rhythmicity, or direction (Koven and Lamm, 1954). Athetoid movements are typically more severe in the upper limbs and involve the skeletal musculature of the distal parts more severely than the proximal (Bucy, 1942). The disorder may involve the muscles of articulation, the face or neck, one extremity, both extremities of one side, both arms, or the trunk (Putnam, 1939). These involuntary movements are known to be extremely variable (Herz, 1931) and are very difficult to quantify. They have been studied by a number of workers, particularly Foerster (1921), Wilson (1925), and Herz (1931). Herz documented his analysis with motion pictures. After studying the films he concluded that athetoid movement contains no synergistic coordinated activity nor is there a regular sequence of movement. Similarities with the actions of grasping (Berger, 1903), piano playing, or relinquishing an object (Wilson,

1 Address for reprints: Clinical Sciences Building, The Prince Henry Hospital, Little Bay, N.S.W., Australia.
1925) have been noted. The movements have been described as vermicular, cramplike, spasmodic, and writhing.

Description of the involuntary movement is considered important in both the diagnosis and classification of athetosis. For example, the American Academy for Cerebral Palsy accepts a classification of cerebral palsy based on motor symptomatology. Athetosis is classified into 12 sub-groups based on a qualitative description of the involuntary movements (Minear, 1956; Phelps, 1956). The importance of classification in athetosis was pointed out by Phelps (1956) with regard to selection of the treatment programme most appropriate for an individual patient. Such a system of classification, however, can be no better than the clinician's ability to describe the involuntary movements. Descriptions based solely on inspection of the patient are probably of doubtful reliability. For example, some authors describe the movements as monotonous (Jakob, 1925), while others emphasize the variability (Herz, 1931); some authors describe them as rhythmical (Lewandowsky, 1910), while others say they are not rhythmical 
(Bostroem, 1939; Koven and Lamm, 1954). Furthermore, precise information about speed, range, and power of involuntary movement is lost in gross descriptive terms such as flailing, writhing, etc.

Before the introduction of the term athetosis by Hammond (1871) almost all abnormal involuntary activity was described as chorea (Herz, 1944). The separation of chorea and athetosis was initially criticized but it has proved to be of clinical value and is retained by modern authors.

Analysis of motion pictures showed that athetoid patients can display quick, jerky movements similar to those in chorea. This gives rise to the more inclusive term choreoathetosis.

The electromyogram (EMG) from athetoid muscles shows an asynchronous discharge of motor units resembling normal voluntary innervation but the opposing muscles do not relax (Putnam, 1939). The peculiar character of athetoid movement is caused by lack of reciprocal inhibition (Hoefer and Putnam, 1940).

Several types of involuntary activity have been observed in the EMG from athetoid muscle (Lindsley, 1936). In some patients there are slow squirming movements of the dystonic type involving whole groups of muscles, in other patients there are oscillations of the limb caused by a series of clonic contractions which persist for a few seconds at a time.

Lindsley (1936) and Hoefer and Putnam (1940) observed grouping of the impulses in the EMG from all cases. The frequency of the groupings varies from 2 to 11 volleys per second. They also described an oscillation of the limb resembling the tremor of Parkinson's disease. Hoefer and Putnam (1940) claimed that tremor coexists with the choreoathetotic movement and that tremor is not a symptom of athetosis in its pure form. This view is supported by the observation that tremor has been seen to persist, while athetosis has been reduced by anterolateral spinal cordotomy (Putnam, 1933).

A quantified description of athetoid movement is required for both clinical and research purposes. For example, after a 12 year programme of assessment of relaxant drugs in cerebral palsy, Denhoff and Holden (1961) concluded that there is an urgent need to use more objective measures to evaluate drug effectiveness.

A recent study of voluntary control of move- ment in athetosis (Neilson, 1974) suggests a $\frac{O}{z}$ method by which involuntary movements may be quantified. The method is attractive because it provides a statistical description of the athetoid movements made during voluntary activity. This allows the possibility of investigating the interaction between voluntary and involuntary activity. Involuntary movements usually subside in sleep and some patients show practically no involuntary activity when they are in a resting state (Koven and Lamm, 1954; Twitchell, 1961). During voluntary activity, however, athetoid movement appears, causing an increase in energy output, sweating and a marked rise in basal metabolism (Putnam, 1939). In severe cases purposive motion can be completely disrupted by athetoid activity.

The purpose of the experiments explained below is to provide a method of measurement and a quantified statistical description of involuntary arm movements about the elbow joint $\stackrel{\omega}{v}$ made by athetotic patients while they are per $_{-}$i forming a sequence of voluntary arm moves ments.

METHOD

The method used for the tracking test was the sam as described previously (Neilson, 1973) and so wit be only briefly re-explained.

The patient sat watching an oscilloscope screen on which he could move a horizontal line up and down by flexion-extension movements of the right arm about the elbow. A $10^{\circ}$ flexion movement caused a $10 \mathrm{~mm}$ upward deflection of the oscilloscope line. A second not so bright and slightly defocused line on the screen was moved up and down in an irregular fashion by the experimenter. The task for the patient was to keep the two lines superimposed on the screen. The positions of both the target and elbowangle lines on the screen were recorded on a 4 channel Grass polygraph. A $10 \mathrm{~mm}$ upward deflection of either line corresponded to a $5 \mathrm{~mm}$ upward deflection of the appropriate polygraph pen. The electromyogram (EMG) and integrated EMG (IEMG) of the biceps brachii muscle were also recorded on the polygraph. The time constant of the filter in the integrator was adjusted to $0 \cdot 16 \mathrm{sec}$. The EMG from both biceps and triceps muscles were amplified and displayed on a 17 in. oscilloscope so they could be monitored and checked for artefact throughout the experiment. The results from 10 athetotic cerebral palsied patients were analysed. 
TECHNIQUE OF ANALYSIS In the previous study the mathematical relationship between the target signal and that portion of the elbow-angle signal coherent with the target signal was computed. The relationship was described graphically by plotting the gain and phase frequency response curves. Statistical descriptions of the target, IEMG, and elbow-angle signals were obtained by computing the auto power spectrum of each signal. With this knowledge the auto power spectra describing both the voluntary and involuntary arm movements can be calculated. To obtain the voluntary movement spectrum the power spectrum of the target signal is multiplied by the square of the gain at each frequency. In mathematical terms the output power spectrum equals the input power spectrum multiplied by the square of the transfer function (Blackman and Tukey, 1958; Jenkins and Watts, 1968). By subtracting the power spectrum of the voluntary movements from that of the total elbow-angle signal, the power spectrum describing the involuntary arm movements is obtained. The power spectra of both the voluntary and involuntary components of the IEMG signal are computed in the same way.

The statement that the spectra describe the voluntary and involuntary activity is not completely accurate. More correctly, the division is between that component of either the elbow-angle or IEMG signal that is coherent with the target signal and that component which is incoherent. This separation, however, parallels very closely the division between voluntary and involuntary activity. Furthermore, athetoid power spectra of elbow-angle and IEMG signals contained a secondary peak which was not present in the power spectra from either spastic patients or normal subjects (Neilson, 1972a, b, 1974). This peak was almost certainly caused by the involuntary activity of athetosis. Thus it seems reasonable that the incoherent power spectra of the elbowangle and IEMG signals provide an objective description of the involuntary activity.

As described above the IEMG signal was obtained by integrating the EMG signal with a time constant of $0.16 \mathrm{sec}$. The EMG signal was full wave rectified and filtered by a single pole low pass filter with a corner frequency of $1 \mathrm{~Hz}$. Consequently, the IEMG power spectrum includes the high frequency attenuation introduced by the filter. The IEMG power spectrum can be corrected for this attenuation by multiplying by the square of the inverse characteristics of the integrating filter. Both the corrected and uncorrected power spectra of the involuntary IEMG activity have been computed in this study.

\section{RESULTS}

Polygraph recordings of target, elbow-angle, IEMG, and EMG signals were recorded during the visual tracking tests (Fig. 1). Inspection of the tracings shows fluctuations and oscillations in the IEMG and elbow-angle signals which are not correlated with the target signal. These fluctuations apparently represent the involuntary activity. The most striking feature in the polygraph recordings is the pattern of bursts in the EMG and IEMG signals. It was present in both flexor and extensor muscles whenever the patient was voluntarily moving the arm but was absent when he was at rest. If the intervals between

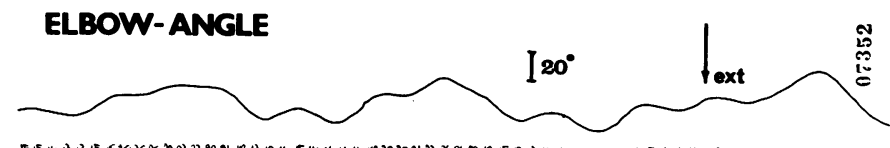

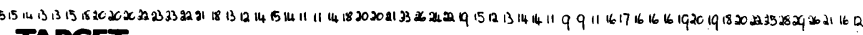
TAREET.
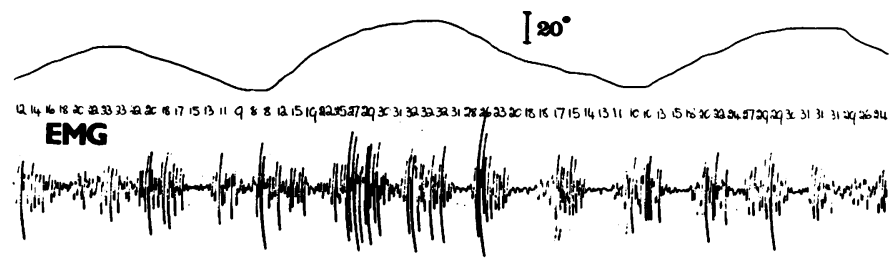

IEMG

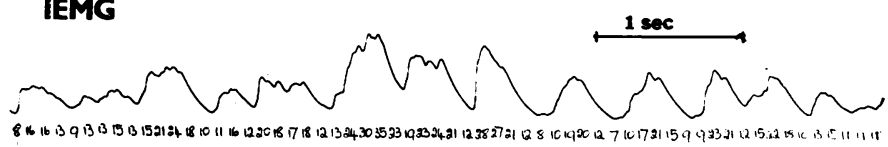

FIG. 1. A typical page of polygraph tracings of elbow-angle, target, EMG, and IEMG signals recorded during $a$ visual tracking test. Involuntary oscillations and fluctuations can be seen in the elbow-angle signal and athetoid action tremor can be seen clearly in both the EMG and IEMG tracings. 
bursts are measured during only a few seconds of recording one gets the impression that the activity is rhythmical at a frequency of about $2.5 \mathrm{~Hz}$. Long-term recording, however, reveals that both the amplitude and frequency of the bursts alter with time and that activity in opposing muscle groups is poorly correlated. The activity is sometimes synchronous, sometimes
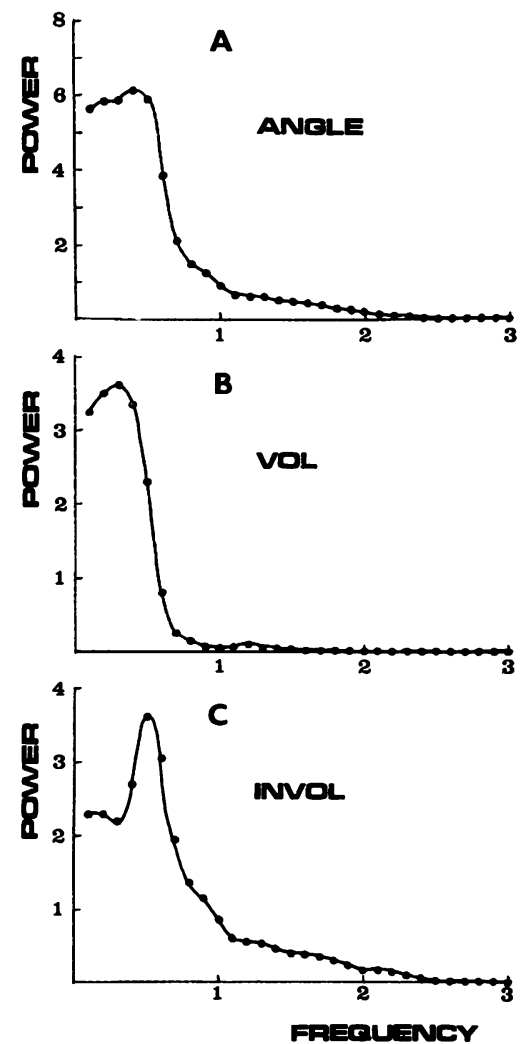

FIG. 2. A. Auto power spectrum of the elbow-angle signal recorded during a visual tracking test. B. Auto power spectrum of the component of the elbow-angle signal that is coherent with the target signal. This is the power spectrum of voluntary arm movement. It diminishes to a negligible value between 1-1.5 Hz. C. Auto power spectrum of the component of the elbow-angle signal that is incoherent with the target signal. This is approximately equivalent to the power spectrum of the involuntary arm movement. It diminishes to a negligible value between $2-3 \mathrm{~Hz}$ and sometimes contains small secondary peaks at $1 \cdot 5-3 \mathrm{~Hz}$ which are not present in this figure. In each spectrum power is plotted in arbitrary units and frequency is plotted in $\mathrm{Hz}$. asynchronous, and often the frequency of bursting is different in opposing muscle groups.

Using the technique of analysis explained above the power spectrum of the elbow angle signal recorded during visual tracking was divided into voluntary and involuntary components (Fig. 2). Voluntary activity was greatest at low frequencies $(<0.2 \mathrm{~Hz})$ but decreased with increasing frequency, becoming negligible by $1-1.5 \mathrm{~Hz}$. The power spectrum of the involuntary movements started at intermediate levels of power at low frequencies, increased with frequency reaching a maximum between $0.3-0.6 \mathrm{~Hz}$
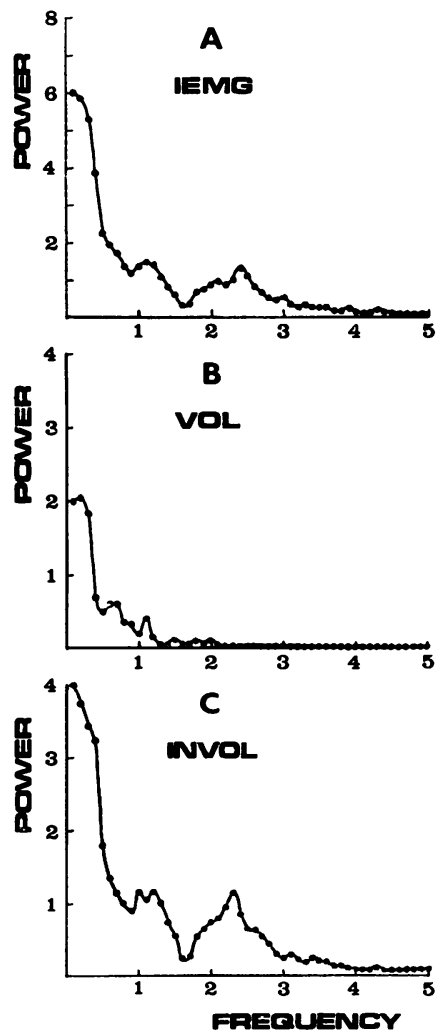

FIG. 3. A. Auto power spectrum of the IEMG signal recorded during a visual tracking test. B. Power spectrum of the component of the IEMG signal that is coherent with the target signal. This is the power spectrum of the voluntary component of the IEMG signal. C. Power spectrum of the component of the IEMG signal that is incoherent with the target signal. In each spectrum power is plotted in arbitrary units and frequency is plotted in $\mathrm{Hz}$. 
and then decreased again. Consequently there was always a peak in the power spectra of the involuntary movements at a frequency $0.3-0.6$ Hz. Power diminished with increasing frequency, reaching a negligible value by $3 \mathrm{~Hz}$. Small secondary peaks could sometimes be seen at the higher frequencies $(1 \cdot 5-3 \mathrm{~Hz})$.

A typical measure of power at $2 \mathrm{~Hz}$ was about 1-2 units (Fig. 2). This corresponds to an average amplitude of elbow-angle change of about $3^{\circ}$ peak to peak. Individual oscillations at $2 \mathrm{~Hz}$ were measured directly from the polygraph tracings with amplitudes as large as $10^{\circ}$ peak to peak. This variation between the mean value and individual measurements indicates that the amplitude of the elbow-angle oscillation at $2 \mathrm{~Hz}$ varied with time. The amplitude of both the elbow-angle oscillation and the bursting in the EMG and IEMG signals could clearly be seen in the polygraph recordings to change with time in an irregular fashion.

The power spectrum of the IEMG signal was also divided into voluntary and involuntary components (Fig. 3). The voluntary IEMG spectrum had a large low frequency peak. It decreased with increasing frequency reaching a negligible value by $1-1.5 \mathrm{~Hz}$. The involuntary IEMG spectrum also had a large low frequency peak but there was a secondary peak as well (Fig. 3C). The secondary peak was caused by the bursting activity in the IEMG signal. The second-

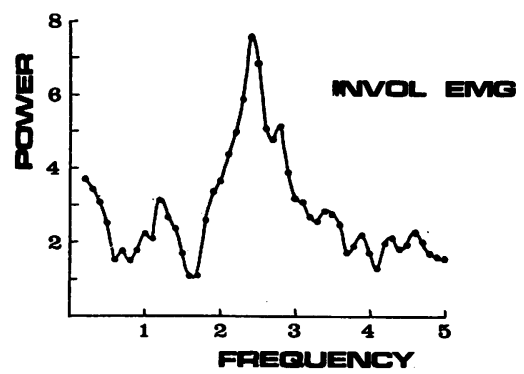

FIG. 4. Auto power spectrum of the involuntary component of the IEMG signal after it has been corrected for the attenuation distortion introduced by the integrating filter. The large amplitude of the secondary peak emphasizes the magnitude of the athetoid action tremor in the electromyogram. The broad base of the peak indicates that the frequency of the tremor varies in the range $1 \cdot 5-4 \mathrm{~Hz}$. ary peak was not sharply tuned as one might expect for rhythmical bursting activity but was broad based and spread over a range of frequencies between $1 \cdot 5-4 \mathrm{~Hz}$. This is consistent with the observation that the bursting frequency varied with time. The shape of the peak indicates that the frequency varied in the range $1 \cdot 5-4 \mathrm{~Hz}$ with a predominant frequency of about $2.5 \mathrm{~Hz}$.

A large amount of high frequency $(>2 \mathrm{~Hz})$ IEMG activity was recorded and this is emphasized by the large peak in the IEMG spectrum after it has been corrected for the attenuation distortion introduced by the integrating filter (Fig. 4). In spite of the magnitude of this activity, only very small elbow angle movements were recorded at these frequencies and this is verified by the small power levels in the elbow-angle power spectra (Fig. 2).

\section{DISCUSSION}

For the most part the fluctuations and oscillations in the elbow-angle signal which cannot be seen in the target signal are caused by the involuntary movements of athetosis. The most striking feature in the polygraph recording (Fig. 1) is the occurrence of bursts of EMG and IEMG activity which may be seen in both agonist and antagonist muscle groups whenever the patient is active. Lindsley (1936) and Hoefer and Putnam (1940) described the grouping of impulses in the EMG as resembling the tremor of Parkinson's disease. The bursts of activity in athetosis, however, are dissimilar to those of the tremor of Parkinson's disease in a number of respects (Andrews et al., 1973a). The broad base of the secondary peak in the involuntary IEMG spectrum (Fig. 4) indicates that the frequency of the EMG burst from biceps in an individual athetotic patient varies over a wide range $(1 \cdot 5-4 \mathrm{~Hz})$, whereas the tremor frequency in Parkinsonism is relatively constant in any one patient in the range 3-7 Hz (Lance et al., 1963). In athetosis tremor is not present at rest but appears during voluntary activity. In Parkinson's disease an alternating tremor is present at rest and usually disappears during activity when it is replaced by an action tremor of higher frequency $(10 \mathrm{~Hz})$ in which the beats of EMG activity are synchronous in antagonistic muscle pairs. In athetosis the EMG activity in opposing muscle groups is 
poorly correlated and the bursts may occur with different frequencies in agonist and antagonist. These differences suggest that a neurological mechanism other than that causing the tremor of Parkinson's disease is operative and the term athetoid action tremor would seem appropriate.

The results of this study oppose the view that tremor is not a symptom of athetosis in its pure form (Hoefer and Putnam, 1940). In fact, it is argued that athetoid action tremor is one of the most outstanding features of athetosis. It is difficult to observe athetoid action tremor by inspection of the patient or by goniometric recording because the movement is filtered out by the abnormal mechanical load on muscle in athetosis (Neilson, 1974).

Putnam's finding that tremor and involuntary writhing movement can be altered independently by anterolateral spinal cordotomy does not imply that tremor is an entity separate from the athetotic syndrome but could indicate that they are generated by different neurological mechanisms.

Apart from athetoid action tremor two other components of involuntary activity are revealed by the power spectrum of the involuntary elbowangle movement. There is a continuous distribution of power across the low frequency band $(<3 \mathrm{~Hz})$ which indicates irregular low frequency involuntary movement. As already discussed, the falling off of the power spectrum towards $3 \mathrm{~Hz}$ is caused by mechanical filtering. Mixed with the irregular movement is a very low frequency $(0 \cdot 3-$ $0.6 \mathrm{~Hz}$ ) rhythmical component which is indicated by a predominant peak in the involuntary elbowangle power spectrum (Fig. 3C). Again, this rhythmical component is difficult to observe on clinical examination of the patient because it is masked by irregular movement. The relative flatness of the elbow-angle power spectrum (Fig. $3 \mathrm{~A})$ illustrates this masking effect. The presence of the low frequency rhythmical component was predicted in a previous paper (Neilson, 1974) because of underdamping measured in the voluntary control of movement in athetosis. This finding suggests that the low frequency $(0 \cdot 3-0 \cdot 6$ $\mathrm{Hz}$ ) rhythmical movement is a transient response of the closed loop voluntary control system excited by rapid voluntary movement. This finding indicates that at least two neurological mechanisms probably contribute to the low frequency involuntary movement of athetosis.

In conclusion, a statistical description of involuntary activity in the biceps muscle of 0 athetotic patients indicates at least three separate components each of which may be caused by a different physiological mechanism. There are low frequency irregular movements which are obvious on clinical examination and are commonly recognized as athetosis. This activity is probably generated in supraspinal centres (Bucy, 1942) and Putnam (1936) claims that it can be eliminated by section of extrapyramidal tracts in the anterolateral quadrant of the spinal cord. There is also an athetoid action tremor at about $2.5 \mathrm{~Hz}$ which can be seen most clearly in the electromyogram. It is quite unlike the tremor of Parkinson's disease and is only present when the patient is voluntarily active. In addition, there is a low frequency rhythmical component caused by an abnormal underdamping measured previously in the closed loop voluntary control of elbov' position.

I am most grateful to Associate Professor J. $W_{\bar{O}}^{\varrho}$ Lance and Professor E. P. George for their support 응 and encouragement and the Spastic Centre of Neve South Wales for Centre Industries Research Scholarship. The diagrams were photographed bse the Department of Medical Illustration, University of New South Wales.

\section{REFERENCES}

Andrews, C. J., Burke, D., and Lance, J. W. (1973ג). The comparison of tremors in normal, parkinsonian and athetotic man. Journal of Neurological Science. (In press.)

Andrews, C. J., Neilson, P. D., and Knowles, L. (1973b). Electromyographic study of the rigidospasticity of athetosis. Journal of Neurology, Neurosurgery, and Psychiatry, 36, 94103.

Berger, A. (1903). Zur Kenntnis der Athetose. Jahrbüch für Psychiatrie und Neurologie, 23, 214-233.

Blackman, R. B., and Tukey, J. W. (1958). The Measurement of Power Spectra. Dover Publications: New York.

Bostroem, A. (1939). Krankheiten des extrapyramidalen Systems. In Handbuch der Inneren Medizin, 3rd edn. Vol. 5, Part 1, pp. 668-710. Edited by G. V. Bergmann and R. Staehelin. Springer: Berlin.

Bucy, P. C. (1942). Neural mechanisms of athetosis and tremor. Journal of Neuropathology, 1, 224-239.

Denhoff, E., and Holden, R. H. (1961). Relaxant drugs in cerebral palsy: 1949-1960. New England Journal of Medicine, 264, 475-480.

Foerster, O. (1921). Zur Analyse und Pathophysiologie der striären Bewegungsstörungen. Zeitschrift fiir die gesamte Neurologie und Psychiatrie, 73, 1-169.

Hammond, W. A. (1871). A Treatise on Diseases of the Nervous System, pp. 654-662. Appleton: New York. 
Herz, E. (1931). Die amyostatischen Unruheerscheinungen. Klinischkinematographische Analyse ihrer Kennzeichen und Begleiterscheinungen. Jahrbüch für Psychologie und Neurologie, 43, 3-182.

Herz, E. (1944). Dystonia. I. Historical review; analysis of dystonic symptoms and physiologic mechanisms involved. Archives of Neurology and Psychiatry, 51, 305-318.

Hoefer, P. F. A., and Putnam, T. J. (1940). Action potentials of muscles in athetosis and Sydenham's chorea. Archives of Neurology and Psychiatry, 44, 517-531.

Jakob, A. (1925). The anatomy, clinical syndromes and physiology of the extrapyramidal system. Archives of Neurology and Psychiatry, 13, 596-620.

Jenkins, G. M., and Watts, D. G. (1968). Spectral Analysis and its Applications. Holden-Day: San Francisco.

Koven, L. J. and Lamm, S. S. (1954). The athetoid syndrome in cerebral palsy. Pediatrics, 14, 181-192.

Lance, J. W., Schwab, R. S., and Peterson, E. A. (1963). Action tremor and the cogwheel phenomenon in Parkinson's disease. Brain, 86, 95-110.

Lewandowsky, M. (1910). Die zentralen Bewegungsstörungen. In Handbuch der Neurologie, Vol. I, part 2, pp. 685772. Edited by M. Lewandowsky. Springer: Berlin.

Lindsley, D. B. (1936). Electromyographic studies of neuromuscular disorders. Archives of Neurology and Psychiatry, 36, 128-157.

Minear, W. L. (1956). A classification of cerebral palsy. Pediatrics, 18, 841-852.

Narabayashi, H., Nagahata, M., Nagao, T., and Shimazu, H.
(1965). A new classification of cerebral palsy based upon neurophysiologic considerations. Confinia Neurologica, 25, 378-392.

Neilson, P. D. (1972a). Speed of response or bandwidth of voluntary system controlling elbow position in intact man. Medical and Biological Engineering, 10, 450-459.

Neilson, P. D. (1972b). Voluntary and reflex control of the biceps brachii muscle in spastic-athetotic patients. Journal of Neurology, Neurosurgery, and Psychiatry, 35, 589-598.

Neilson, P. D. (1974). Voluntary control of arm movement in athetotic patients. Journal of Neurology, Neurosurgery, and Psychiatry, 37, 162-170.

Neilson, P. D., and Andrews, C. J. (1973). A comparison of the tonic stretch reflex in athetotic patients during rest and voluntary activity. Journal of Neurology, Neurosurgery, and Psychiatry, 36, 547-554.

Phelps, W. M. (1956). Classification of athetosis with special reference to the motor classification. American Journal of Physical Medicine, 35, 24-31.

Putnam, T. J. (1933). Treatment of athetosis and dystonia by section of extrapyramidal motor tracts. Archives of Neurology and Psychiatry, 29, 504-521.

Putnam, T. J. (1939). The diagnosis and treatment of athetosis and dystonia. Journal of Bone and Joint Surgery, 21, 948-957.

Twitchell, T. E. (1961). The nature of the motor deficit in double athetosis. Archives of Physical Medicine, 42, 63-67.

Wilson, S. A. K. (1925). Some disorders of motility and of muscle tone, with special reference to the corpus striatum. Lancet, 2, 215-219. 